The Astrophysical Journal SupPlement SerIES, 92:425-428, 1994 June

(c) 1994. The American Astronomical Society. All rights reserved. Printed in U.S.A.

\title{
DIFFUSE GALACTIC CONTINUUM EMISSION MEASURED BY COMPTEL AND THE COSMIC-RAY ELECTRON SPECTRUM
}

\author{
A. W. STRONG, R. Diehl, V. SChÖNFELDER, M. VARENDORFF, AND G. YOUSSEFI \\ Max-Planck Institut für extraterrestrische Physik, Postfach 1603, D-85740 Garching, Germany \\ H. Bloemen, W. HeRmSEN, AND C. DE VRIES \\ SRON-Leiden, P.B. 9504, NL-2300 RA Leiden, The Netherlands \\ D. MORRIS AND J. G. STACY \\ University of New Hampshire, Institute for the Study of Earth, Oceans, and Space, Durham, NH 03824 \\ AND \\ K. BENNETT \\ Astrophysics Division, Space Science Department of ESA, ESTEC, 2200 AG Noordwijk, The Netherlands \\ Received 1993 April 12; accepted 1993 July 15
}

\begin{abstract}
Diffuse galactic continuum $\gamma$-ray emission in the $0.75-30 \mathrm{MeV}$ range from the inner Galaxy has been studied using data from COMPTEL on the Compton Gamma-Ray Observatory. Observations of the inner Galaxy from the Sky Survey have been used. The imaging properties of COMPTEL enable spatial analysis of the $\gamma$-ray distribution using model fitting. A model based on atomic and molecular gas distributions in the Galaxy has been used to derive the emissivity spectrum of the $\gamma$-ray emission and this spectrum is compared with theoretical estimates of bremsstrahlung emission from cosmic-ray electrons.
\end{abstract}

Subject headings: diffuse radiation — gamma rays: observations

\section{INTRODUCTION}

The diffuse continuum $\gamma$-ray emission from the Galactic plane at $\mathrm{MeV}$ energies is believed to originate mainly in bremsstrahlung interactions of cosmic-ray electrons with interstellar gas. Measurement of the $\gamma$-ray spectrum at low energies therefore provides important constraints on the spectrum and propagation of cosmic-ray electrons. This information is complementary to results from radio synchrotron and direct measurements of electrons. Together with cosmic-ray composition studies of nucleons the aim is to gain a consistent picture of cosmic-ray origin and propagation including such questions as the nature of the sources, the size of the propagation region (e.g., existence of a "halo") and the relative roles of diffusion and convection. Because of the large energy losses due to ionization, low-energy electrons are especially sensitive to Galactic propagation effects. Energy losses are related to the gas distribution at low energies (ionization and bremsstrahlung) and to magnetic fields and radiation density (synchrotron and inverse Compton emission) at high energies; it follows that the electron spectrum is sensitive to the spatial properties of the propagation process.

Observations at $\mathrm{MeV}$ energies are difficult and until now have mainly been made with nonimaging detectors which give a wide-angle average of the emission to be measured. The COMPTEL instrument on the Compton Gamma-Ray Observatory has sufficient imaging capability to allow a sensitive measurement of the diffuse radiation. Preliminary mapping of the Galactic plane (see Bloemen et al. 1994) shows that the ridge can be detected; the energy spectrum may then be determined by model fitting. The present approach to COMPTEL data analysis has been largely influenced by that for the $C O S B$ data (Bloemen et al. 1986; Strong et al. 1988); in that work it was found possible to separate the components of diffuse emission associated with atomic and molecular gas in the Galaxy, and further to determine the large-scale emissivity gradient; COMPTEL data do not yet allow such a detailed analysis and the modeling is relatively simple by comparison. However the component separation should become possible as the database expands to cover the full Galactic plane with good exposure.

\section{OBSERVATIONS AND METHOD}

The principles of COMPTEL measurements are given in detail by Schönfelder et al. (1993). For each incident photon detected by the instrument, the energy deposits and interaction locations in the upper and lower detectors are measured. The Compton scatter angle $\bar{\varphi}$ is computed from the measured energy deposits, and the photons are binned in a three-dimensional data space consisting of the scatter direction and $\bar{\varphi}$. The response of the instrument in this data space to a given incident $\gamma$-ray intensity distribution is computed on the basis of the knowledge of the instrument configuration as a function of time, the pre-launch calibration and simulations. Hence, given a parameterized model of the sky, the values of the parameters can be determined by model fitting in the data space described above.

We assume the continuum radiation originates in cosmicray interactions with interstellar gas, and therefore use the relation

$$
I_{\gamma}=\frac{q}{4 \pi} N_{\mathrm{H}}+I_{B}
$$


where $I_{\gamma}$ is the $\gamma$-ray intensity $\left(\mathrm{cm}^{-2} \mathrm{~s}^{-1}\right), q$ is the $\gamma$-ray emissivity per $\mathrm{H}$ atom, $N_{\mathrm{H}}$ is the total hydrogen gas column density and $I_{B}$ is a celestial background term. $N_{\mathrm{H}}$ is taken from $\mathrm{H}$ I and $\mathrm{CO}$ surveys, assuming that the molecular hydrogen column density is related to the integrated $\mathrm{CO}$ temperature by

$$
\frac{N_{\mathrm{H}_{2}}}{W_{\mathrm{CO}}}=2.3 \times 10^{20} \text { molecules } \mathrm{cm}^{-2}\left(\mathrm{~K} \mathrm{~km} \mathrm{~s}^{-1}\right)^{-1}
$$

as determined from $C O S B$ data by Strong et al. (1988) and supported by various other methods (Schloerb et al. 1990; Kent et al. 1991).

The free parameters of the model are $q, I_{B}$, and the instrumental background level. Since the instrumental background is rather high and not known a priori, its treatment is critical in obtaining a reliable result. We use high-latitude observations as the basis for our estimates, assuming that the shape of this background is the same in the instrument system for all observations. To obtain a good estimate of the average background shape several high-latitude observations were summed and the result slightly smoothed. The absolute level of instrumental background was determined by the fitting in data space.

\section{GAMMA-RAY EMISSIVITY SPECTRUM}

Table 1 summarizes the 10 observations used in this work. The data for the observations were combined by adding the counts and computing the effective response for the combined data set.

The fit to equation ( 1 ) in four energy ranges gave compatible values for $q$ when each of the observations was analyzed separately; therefore a fit to the combined data set is justified and is used in what follows. We can conclude that the Galactic continuum radiation was indeed detected and the emissivities can be reasonably interpreted in terms of emission from the interstellar medium. Table 2 summarizes the results.

Figure 1 shows the emissivity spectrum of the inner Galaxy based on the present results and also values obtained from the application of a similar method fitting $C O S B$ data to the same gas model Strong et al. (1988). The COMPTEL points are consistent with a spectral index of -2.0 and this slope is used to convert the values for each energy range to a differential spectrum in this plot. Errors on the COMPTEL points are estimated to be about $30 \%$, including statistical and systematic errors. The spectrum is in agreement with a smooth connec-

TABLE 1

SUMMARY OF COMPTEL OBSERVATIONS USED

\begin{tabular}{|c|c|c|c|}
\hline Observation & Target & $l$ & $b$ \\
\hline 2.0 & Cyg X-1 & $73^{\circ}$ & $+3^{\circ}$ \\
\hline 5 . & Galactic Center & 0 & -4 \\
\hline$\ldots \ldots$ & Cyg X-3 & 70 & -8 \\
\hline $7.5 \ldots \ldots \ldots \ldots$ & $\mathrm{Gal} 025-14$ & 25 & -14 \\
\hline $8 \ldots \ldots \ldots \ldots$ & Vela PSR & 263 & -6 \\
\hline $13.0 \ldots \ldots \ldots \ldots$ & Gal $025-14$ & 25 & -14 \\
\hline $14 \ldots \ldots \ldots \ldots$ & Eta Car & 285 & -1 \\
\hline $20 \ldots \ldots \ldots \ldots$ & SS 433 & 40 & +1 \\
\hline $23 \ldots \ldots \ldots \ldots$ & Cir X-1 & 322 & +3 \\
\hline $34 \ldots \ldots \ldots \ldots$ & Cas A & 109 & -2 \\
\hline
\end{tabular}

TABLE 2

INNER GALAXY EMISSIVITIES FOR INTEGRAL ENERGY RANGES

\begin{tabular}{|c|c|c|c|c|}
\hline Energy range (MeV) & $0.75-1.0$ & $1.0-3.0$ & $3.0-10.0$ & $10.0-30.0$ \\
\hline$q / 4 \pi\left(10^{-25} \mathrm{sr}^{-1} \mathrm{~s}^{-1}\right)$ & 2.6 & 4.8 & 1.6 & 0.41 \\
\hline
\end{tabular}

tion to the $\operatorname{COS} B$ spectrum. Although our values represent strictly an upper limit since there may be some contribution from point sources in the Galactic plane, the continuity with the higher energy results suggests that we are in fact mainly detecting diffuse emission at least in the higher energy ranges. This point is discussed further in the context of the theoretical modeling (§ 4$)$.

In order to compare with other experimental results for the Galactic center region, where the results are normally given as a flux per radian integrated over latitude, we use, for the COMPTEL and $C O S B$ intensities, the quantity

$$
J_{\gamma}=\frac{q}{4 \pi} \int_{-20^{\circ}}^{+20^{\circ}} N_{\mathrm{H}} d b
$$

averaged over $-30^{\circ}<l<+30^{\circ}$. Figure 2 shows the longitude dependence of $\int_{-20^{\circ}}^{+20^{\circ}} N_{\mathrm{H}} d b$ for reference. Figure 3 shows a compilation of results for the flux in the inner Galaxy; note that such a comparison is at best approximate because of the very different angular responses of the various instruments and the fact that the observations refer to different regions of the Galactic plane. Nevertheless the COMPTEL result is consistent with the overall spectrum; it suggests, however, that the true spectrum is about a factor of 2 lower than the "best guess" of Gehrels \& Tueller ( 1992). The difficulty of comparison underlines the need for a uniform analysis method for different instruments; note that the approach used here could be applied to data from other instruments and this would allow direct comparison of results (as is the case for the COMPTEL and $\operatorname{COS} B$ analysis shown in Fig. 1).

\section{INTERPRETATION: SPECTRUM OF COSMIC-RAY ELECTRONS}

The low-energy interstellar electron spectrum is not directly measurable (due to solar modulation) so we have to rely on

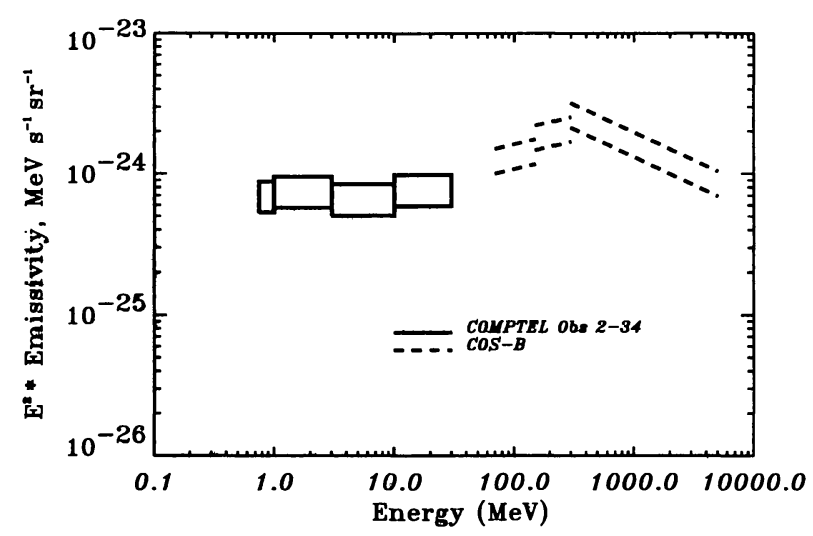

FIG. 1.-Emissivity spectrum of the inner Galaxy derived from COMPTEL data together with values from a similar analysis of $\operatorname{COS} B$ data (Strong et al. 1988). 


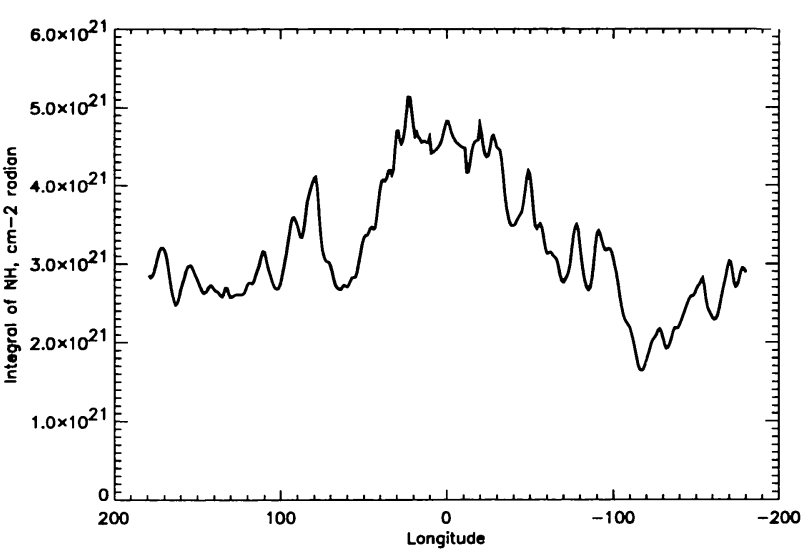

FIG. 2.-Total gas column density $\left(\mathrm{H}\right.$ I and $\mathrm{H}_{2}$ ) from $21 \mathrm{~cm}$ and $\mathrm{CO}$ surveys integrated over $-20^{\circ}<b<+20^{\circ}$.

model calculations to obtain predictions of the $\gamma$-ray emissivity from bremsstrahlung. Spectra for the homogeneous ("leaky-box") model of cosmic-ray propagation have been calculated by Ip \& Axford (1985) and Skibo \& Ramaty (1993) for particular choices of model parameters. A typical set of parameters which fits the cosmic-ray nucleon composition data (Webber 1990) is: mean path length $\left(X_{\mathrm{H}}=\beta c \rho \tau\right)=10.8 \beta$ $\mathrm{g} \mathrm{cm}^{-2}$ for $E_{e}<4 \mathrm{GeV},=24.9 E^{-0.6} \mathrm{~g} \mathrm{~cm}^{-2}$ for $E_{e}>4 \mathrm{GeV}$. For a mean cosmic-ray lifetime $\tau=2.5 \times 10^{7}$ yr this implies a mean gas density of $\rho=0.3 \mathrm{~cm}^{-3}$. The shape of the spectrum over the $1-1000 \mathrm{MeV}$ range is effectively determined by $X_{\mathrm{H}}$ which fixes the energy losses by ionization and bremsstrahlung. We have therefore computed spectra for various $X_{\mathrm{H}}$ to illustrate to what extent the observations constrain this parameter (Fig. 4). The electron injection spectrum is assumed to have a constant index of -2.4 (Webber 1983); the interstellar spectrum is normalized at $1 \mathrm{GeV}$ to the value derived from an analysis of $\operatorname{COS} B$ data (Strong 1985).

The resulting $\gamma$-ray spectrum is shown in Figure 5; we have added to the bremsstrahlung the $\pi^{\circ}$ emissivity from Dermer (1986). The value of $X_{\mathrm{H}}(<4 \mathrm{GeV})$ which best fits the data is 8 $\mathrm{g} \mathrm{cm}^{-2}$ which is in good agreement with the value derived from composition data. However, although the average level (1-30

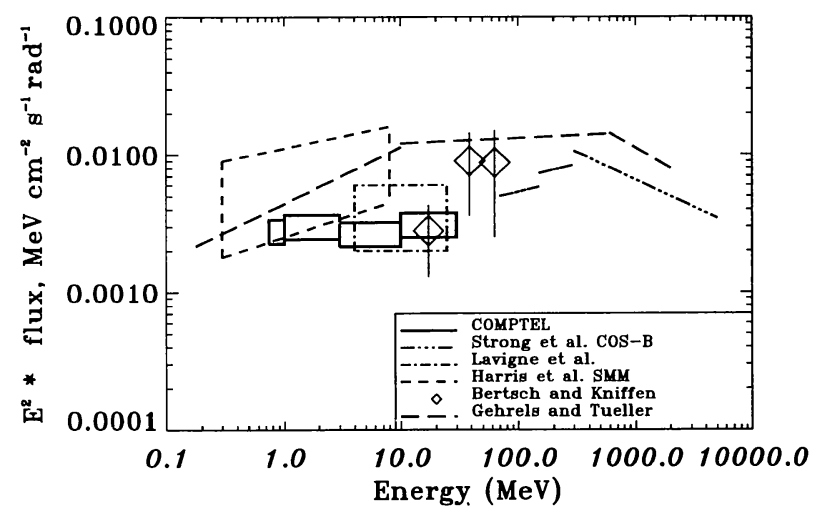

FIG. 3.-Compilation of flux measurements for inner Galaxy. Fluxes are integrated over latitude.

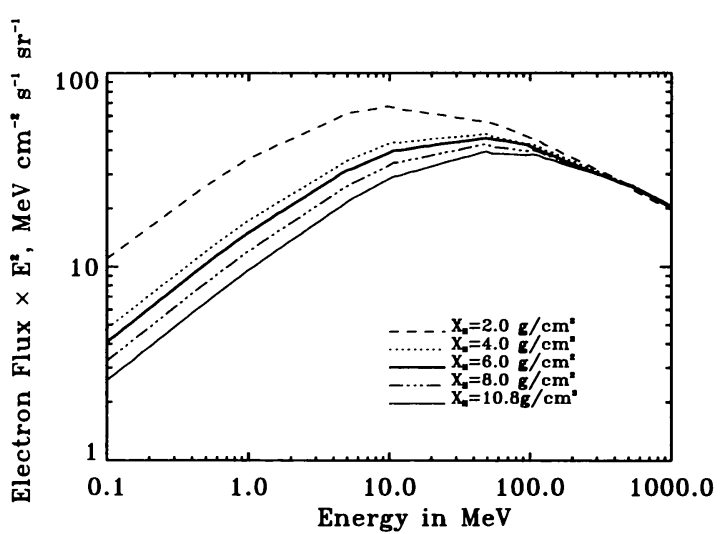

FIG. 4.-Electron spectra for leaky-box models for various values of grammage $\left(X_{\mathrm{H}}\right)$. Spectra are normalized at $1 \mathrm{GeV}$ to the value derived from $\operatorname{COS} B \gamma$-ray data (Strong 1985).

$\mathrm{MeV})$ is consistent with this prediction, the observed spectral shape is somewhat steeper $\left(E^{-2}\right)$; the expected spectral flattening at low energies due to ionization losses is not apparent, although the measurement errors are large enough that the $8 \mathrm{~g}$ $\mathrm{cm}^{-2}$ spectrum cannot definitely be excluded. A possible explanation for the steeper spectrum is that the low-energy emission has a significant contribution from unresolved point-sources which are interpreted as diffuse emission in our model fitting as suggested by the preliminary COMPTEL imaging results (Bloemen et al. 1994). If this is so then our emissivities represent only upper limits on the true values. If bremsstrahlung does indeed dominate this would imply an upturn in the electron spectrum at low energies. Another possibility is inverse Compton emission which has a steeper spectrum and may be important at these energies (see, e.g., Bloemen 1985; Youssefi \& Strong 1991).

\section{CONCLUSIONS}

The present results were derived using only a small part of the COMPTEL Sky-Survey, and already significant constraints can be placed on the interstellar electron spectrum. The analysis will be extended to the entire Galactic plane, and on the basis of the present analysis a further improvement in

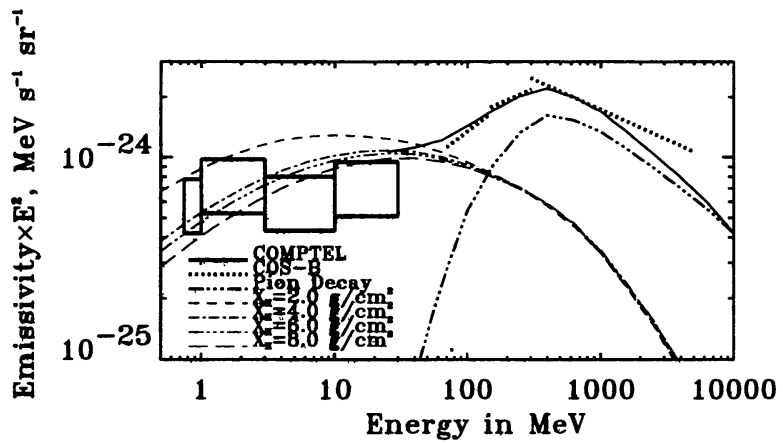

FIG. 5.-Predicted $\gamma$-ray emissivity from bremsstrahlung based on electron spectra (Fig. 4) for various $X_{\mathrm{H}}$, compared with COMPTEL and $\operatorname{COS} B$ results. The solid line above $10 \mathrm{MeV}$ is the sum of bremsstrahlung and $\pi^{\circ}$ emission. 
our knowledge of the low-energy $\gamma$-ray continuum can be expected. The question of the interpretation of the spectral slope, which does not clearly show the behavior expected from ionization losses of electrons, will have to be studied further and the possibility of other components such as unresolved point sources and inverse-Compton emission investigated. The separation into components related to atomic and molecular gas in the Galaxy, as was done for $\operatorname{COS} B$, should be possible with the full Sky Survey database. Combination with complementary OSSE and EGRET results will then be an important step in unravelling the components of the diffuse $\gamma$-ray emission from the Galaxy.

The $C G R O$ project has been supported by DARA under the grant 50 QV 9096 (MPE) and by NASA under grant NAS526645 (UNH).
Bertsch, D. L., \& Kniffen, D. A. 1983, ApJ 270, 305

Bloemen, H. 1985, A\&A, 145, 391

Bloemen, H., et al. 1986, A\&A, 154, 25

Bloemen, H., et al. 1994, ApJS, 92, 419

Dermer, C. D. 1986, A\&A, 157, 223

Gehrels, N., \& Tueller, J. 1992, ApJ, 407, 597

Harris, M. J., et al. 1990, ApJ, 362, 135

Ip, W.-H., \& Axford, W. I. 1985, A\&A, 149, 7

Kent, S. M., et al. 1991, ApJ, 378, 131

Lavigne, J. M., et al. 1986, ApJ, 308, 370

Schönfelder, V., et al. 1993, ApJS, 80, 657

\section{REFERENCES}

Schloerb, F. P., et al. 1990, ApJ, 362, 147

Skibo, J. G., \& Ramaty, R. 1993, A\&AS, 97, 145

Strong, A. W. 1985, Proc. 19th Internat. Cosmic-Ray Conf. (La Jolla), 1, 333

Strong, A. W., et al. 1988, A\&A, 207, 1

Webber, W. R. 1983, in Composition and Origin of Cosmic Rays, ed. M. M. Shapiro (Dordrecht: Reidel), 83

1990, Proc. 21st Internat. Cosmic-Ray Conf. (Adelaide), 3, 393

Youssefi, G., \& Strong, A. W. 1991, Proc. 22d Internat. Cosmic-Ray Conf. (Adelaide), 1, 129 